\title{
Principais erros encontrados em radiografias descartadas na clínica escola de uma instituição de ensino superior
}

\author{
Main errors found in radiographs discarded in the school clinic of an institution of higher \\ education \\ Principales errores encontrados en las radiografías descartadas en la clínica escolar de una \\ institución de educación superior
}

Recebido: 05/01/2021 | Revisado: 05/01/2021 | Aceito: 08/01/2021 | Publicado: 09/01/2021

Filipe de Oliveira Lima ORCID: https://orcid.org/0000-0001-6652-3101 Universidade Federal de Campina Grande, Brasil

E-mail: filipelimaoliveirava@outlook.com

Luciana Ferraz Gominho

ORCID: https://orcid.org/0000-0002-7881-0316 Universidade Federal da Paraíba, Brasil E-mail: fggf.end@gmail.com

Elizandra Silva da Penha

ORCID: https://orcid.org/0000-0001-6264-5232 Universidade Federal de Campina Grande, Brasil E-mail: elizandrapenha@hotmail.com

Manuella Santos Carneiro Almeida ORCID: https://orcid.org/0000-0002-5849-6972 Universidade Federal de Campina Grande, Brasil

E-mail: manuellacarneiro@hotmail.com

Maria Kaline Romero Teodoro

ORCID: https://orcid.org/0000-0002-6482-0628 Faculdade Escritor Osman da Costa Lins, Brasil E-mail: kaline_rote@hotmail.com

Thallita Alves dos Santos ORCID: https://orcid.org/0000-0002-7100-5023 Universidade Federal de Campina Grande, Brasil E-mail: thallita_28@hotmail.com

Mateus Araújo Andrade ORCID: https://orcid.org/0000-0003-3769-2330 Universidade Federal de Campina Grande, Brasil E-mail: mateusewo@outlook.com

Quemuel Pereira da Silva ORCID: https://orcid.org/0000-0002-0093-6322 Universidade Federal de Campina Grande, Brasil E-mail: quemuelpereira7@gmail.com

Maria Tays Pereira Santana ORCID: https://orcid.org/0000-0002-6503-7117 Universidade Federal de Campina Grande, Brasil E-mail: taayssantana@gmail.com

Paula Lima Nogueira

ORCID: https://orcid.org/0000-0001-7425-3201

Universidade Federal de Campina Grande, Brasil

E-mail: paulalimanogueira@hotmail.com

Amanda da Silva Araújo ORCID: https://orcid.org/0000-0003-1034-4459 Universidade Federal de Campina Grande, Brasil E-mail: mandiaraujo55@gmail.com

Maria Vitória Oliveira Dantas ORCID: https://orcid.org/0000-0001-7663-3793 Universidade Federal de Campina Grande, Brasil E-mail: mvitoria.od@hotmail.com

\section{Resumo}

A existência de falhas, sejam elas provocadas durante a exposição ou no processamento radiográfico, resulta em imagens inadequadas que podem interferir no diagnóstico e no tratamento do paciente. O objetivo do presente estudo é fornecer informações sobre os principais tipos de erros cometidos pelos acadêmicos do curso de odontologia e as 
eventuais causas desses erros em radiografias periapicais. É um estudo do tipo transversal, observacional, com abordagem indutiva e procedimento comparativo, estatístico e descritivo. A coleta de dados foi realizada a partir da análise das radiografias descartadas pelos estudantes por erros radiográficas que inviabilizaram a sua utilização. Os erros foram categorizados em erros de técnica radiográfica e erros de processamento radiográfico. No total foram coletadas 114 radiografias as quais faram analizadas por 3 cirurgiões-dentistas. Os erros de técnica radiográfica foram os mais encontrados, correspondendo a $81,58 \%$ da amostra total. Já os erros no processamento radiográficos foram de $18,42 \%$. Dentre esses, o enquadramento com cortes de dentes foi o erro mais encontrado (35,9\%), seguido pela subexposição $(21,05 \%)$, superexposição $(14,04 \%)$ e erro na angulagem vertical $(9,65 \%)$. Em menores percentuais foram encontradas radiografias parcialmente reveladas $(6,14 \%)$, radiografias manchadas $(5,26 \%)$, películas amareladas $(5,26 \%)$, aparecimento de meia lua $(1,35 \%)$ e riscadas $(1,35 \%)$. Pode-se concluir que as principais causas desses erros o posicionamento inadequado do paciente e do aparelho de raios X, radiografias que passaram pouco ou muito tempo em contato com as soluções reveladoras, manuseio inadequado da película após a revelação, armazenamento inapropriado, além de soluções com tempo de uso prolongado indicando esgotamento químico.

Palavras-chave: Odontologia; Ensino; Radiografia dentária; Erros de diagnóstico; Controle de qualidade.

\begin{abstract}
The existence of faults, whether caused during exposure or in radiographic processing, results in inappropriate images that can interfere with the diagnosis and treatment of the patient. The purpose of this study is to provide information on the main types of errors made by dentistry students and the possible causes of these errors on periapical radiographs. It is a cross-sectional, observational study, with an inductive approach and a comparative, statistical and descriptive procedure. Data collection was performed based on the analysis of radiographs discarded by students due to radiographic errors that made their use unfeasible. The errors were categorized into radiographic technique errors and radiographic processing errors. In total, 114 radiographs were collected and analyzed by 3 dentists. The radiographic technique errors were the most found, corresponding to $81.58 \%$ of the total sample. The errors in radiographic processing were $18.42 \%$. Among these, framing with tooth cuts was the most commonly found error $(35.9 \%)$, followed by under-exposure $(21.05 \%)$, over-exposure $(14.04 \%)$ and vertical angle error $(9.65 \%)$. In smaller percentages, partially revealed radiographs (6.14\%), stained radiographs $(5.26 \%)$, yellowish films $(5.26 \%)$, appearance of a half moon (1.35\%) and streaked (1.35\%) were found ). It can be concluded that the main causes of these errors are the inadequate positioning of the patient and the X-ray apparatus, radiographs that have spent little or long time in contact with the processing solutions, improper handling of the film after processing, inappropriate storage, in addition to solutions with prolonged use time indicating chemical depletion.
\end{abstract}

Keywords: Dentistry; Teaching; Dental radiography; Diagnostic errors; Quality control.

\title{
Resumen
}

La existencia de fallas, ya sea causadas durante la exposición o en el procesamiento radiográfico, da como resultado imágenes inapropiadas que pueden interferir con el diagnóstico y el tratamiento del paciente. El propósito de este estudio es proporcionar información sobre los principales tipos de errores cometidos por los estudiantes de odontología y las posibles causas de estos errores en las radiografías periapicales. Es un estudio transversal, observacional, con un enfoque inductivo y un procedimiento comparativo, estadístico y descriptivo. La recopilación de datos se realizó en base al análisis de radiografías descartadas por los estudiantes debido a errores radiográficos que hicieron inviable su uso. Los errores se clasificaron en errores de técnica radiográfica y errores de procesamiento radiográfico. En total, 114 dentistas recolectaron y analizaron 114 radiografías. Los errores de la técnica radiográfica fueron los más encontrados, correspondiendo al $81.58 \%$ de la muestra total. Los errores en el procesamiento radiográfico fueron del $18,42 \%$. Entre estos, el encuadre con cortes de dientes fue el error más comúnmente encontrado (35.9\%), seguido de la subexposición (21.05\%), la sobreexposición (14.04\%) y el error de ángulo vertical $(9.65 \%)$. En porcentajes más pequeños, se encontraron radiografías parcialmente reveladas $(6,14 \%)$, radiografías teñidas $(5,26 \%)$, películas amarillentas $(5,26 \%)$, aparición de media luna $(1,35 \%)$ y rayas $(1,35 \%)$. Se puede concluir que las causas principales de estos errores son el posicionamiento inadecuado del paciente y el aparato de rayos X, radiografías que han pasado poco o mucho tiempo en contacto con las soluciones de procesamiento, manejo inadecuado de la película después del procesamiento, almacenamiento inapropiado, además de soluciones con tiempo de uso prolongado que indica agotamiento químico.

Palabras clave: Odontología; Enseñanza; Radiografía dental; Errores diagnósticos; Control de calidad.

\section{Introdução}

A radiografia é um dos muitos exames complementares disponíveis para viabilizar o estabelecimento de diagnóstico no ramo da Odontologia, agregando informações aos dados clínicos e possibilitando o reconhecimento de eventuais processos patológicos. Para que as informações contidas nas radiografias possuam relevância clínica para o fechamento do diagnóstico é 
fundamental que a técnica utilizada, o processamento e o armazenamento tenham sido realizados de maneira adequada (Silva et al., 2016).

As particularidades da radiografia são tidas como uma instância de caráter objetivo pelos profissionais, onde para isso, somam-se uma gama de fatores como a distorção, contraste, densidade, nitidez e correto enquadramento da área interessada. Portanto, é de suma importância que sejam seguidos da forma correta toda a técnica de tomada e processamento da imagem e do filme radiográfico (Carvalho et al., 2009).

O cirurgião-dentista tem o dever de aumentar a qualidade do exame radiográfico, minimizando o risco atribuído à exposição radiográfica, observando o conceito "ALARA" (As Low As Reasonably Achievable) que preconiza uma exposição radiográfica com a menor dose possível de radiação (Tosoni, Campos \& Silva, 2013). Tendo em vista que erros na técnica utilizada acabam por acarretar repetidas incidências radiográficas aos pacientes com exposições desnecessárias. Além disso, aumentam o risco ao meio ambiente. Antunes (2017) afirma que os resíduos de radiografias têm uma grande componente poluidora do ambiente e prejudicial à saúde humana devido a sua composição química, aos métodos de revelação e recuperação de prata utilizados e à radiação que os aparelhos de radiologia emanam.

Para o profissional da odontologia, os exames radiológicos intrabucais são de longe os mais minudentes, com isso, é desejável que o profissional tenha começado seu contato com a radiologia odontológica ainda quando graduando, onde durante esse tempo é pertinente que haja uma autoavaliação buscando pontos que podem ser aperfeiçoados, objetivando saber como o exame radiográfico pode ser proveitoso ao máximo (Carvalho et al., 2009).

Diante do exposto, o presente estudo objetivou avaliar os erros técnicos em radiografias intrabucais, realizadas por acadêmicos de odontologia, bem como apontar as suas possíveis causas. Com base nos dados obtidos foi plausível explanar a situação para o possível desenvolvimento de estratégias para otimização dos exames radiográficos na clínica odontológica e consequente redução no número de tomadas radiográficas, diminuindo assim os efeitos nocivos ao paciente e ao meio ambiente.

\section{Metodologia}

\subsection{Tipo de Estudo}

Este estudo é uma pesquisa descritiva, com finalidade básica e estratégica, de abordagem mista (quantitativa e qualitativa) sendo transversal, observacional, com enfoque indutivo e procedimento comparativo-estatístico de acordo com as variáveis obtidas, adotando como estratégia de coleta de dados a análise das radiografias intrabucais descartadas pelos estudantes acadêmicos (Pereira et al., 2018).

\subsection{Universo e Amostra}

O universo foi composto pelo número de radiografias encontradas nos recipientes de descarte para filmes radiográficos presentes na Clínica Escola do Curso de Odontologia da Universidade Federal de Campina Grande, no segundo semestre do ano de 2019 e em todas as disciplinas clínicas que funcionaram durante esse período.

\subsection{Critérios de Inclusão}

Foram incluídas no estudo todas as radiografias periapicais encontradas nos recipientes de descartes presentes nas clínicas escola e que foram realizadas pelos acadêmicos do curso de odontologia nos períodos da manhã e da tarde. Foram descartadas as radiografias consideradas insatisfatórias para o uso no planejamento clínico diante de algum tipo de erro cometido na obtenção da imagem. 


\subsection{Critérios de Exclusão}

Foram excluídas do estudo as radiografias intrabucais que não foram descartadas no local adequado, além daquelas que se encontravam muito deterioradas por motivo de mau armazenamento durante o descarte dificultando a identificação dos erros de técnica.

\subsection{Coleta de Dados}

As radiografias foram recolhidas durante período de atendimento clínico das disciplinas do curso de Odontologia por 1 semestre letivo, o que compreendeu aproximadamente 3 meses de coleta de material. As coletas foram coletadas após o térmico de todas as atividades clínicas de cada dia, eram devidamente armazenadas em recipiente hermeticamente fechado e fora do alcance de luz e calor. Ao final do período de coleta foi realizada uma inspeção individual de cada película e todas foram catalogadas em fichas radiográficas. Em seguida, a análise das radiografias foi realizada por dois examinadores especialistas em radiologia, previamente calibrados entre si (Teste Kappa) com auxílio de um negatoscópio, uma lupa e em sala escura. Vale ressaltar que, previamente, foi realizado um estudo piloto com 10 radiografias buscando validar o instrumento de análise da pesquisa.

\subsection{Análise dos Dados}

Para a análise das radiografias foram adotados os seguintes critérios avaliativos de referência para o estudo:

-Erros no momento da tomada radiográfica:

a) $\mathrm{O}$ enquadramento: sendo ainda subdividido em posicionamento errôneo do filme radiográfico, inclusos aqui os filmes com o eixo contrário longo, dente ou parte dele cortados na área radiografada e o surgimento de fenômeno denominado de meia lua. b) A angulagem: dividida em dois grupos, a angulagem vertical (encurtamento e/ou alongamento) e angulagem horizontal.

c) O tempo de exposição: exames radiográficos com subexposição (claras) ou superexposição (escuras), de forma que não era possível obter uma leitura adequada das imagens radiológicas formadas.

d) Radiografias tremidas ou borradas: exames radiográficos sem contornos nítidos em decorrência da movimentação do filme durante a realização do exame.

e) Filme exposto pelo lado da lamínula de chumbo: exames radiográficos feitos pela face contrária a face sensível.

-Erros nas etapas do processamento radiológico:

a) Radiografias manchadas

b) Radiografias amareladas

c) Radiografias riscadas

d) Radiografias reveladas parcialmente

e) Radiografias com impressões digitais

\section{Resultados}

Durante o período de tempo determinado foram recolhidas 114 radiografias impróprias para análise durante o tratamento odontológico. Verificou-se que os erros de técnica radiográfica foram os mais evidenciados, correspondendo a $81,58 \%$ dos erros encontrados. Já os relacionados ao processamento radiográfico foram da ordem de $18,42 \%$ do total de radiografias analisadas. Em específico, o enquadramento com cortes na imagem de um dente foi o erro mais encontrado (35,9\%), sendo seguido pela subexposição $(21,05 \%)$, superexposição $(14,04 \%)$ e erro na angulagem vertical $(9,65 \%)$. Em menores percentuais foram encontradas radiografias parcialmente reveladas $(6,14 \%)$, radiografias manchadas $(5,26 \%)$, películas amareladas $(5,26 \%)$, enquadramento com aparecimento de meia lua $(1,35 \%)$ e riscadas $(1,35 \%)$. 


\section{Discussão}

O exame radiográfico constitui um importante exame complementar de diagnóstico e parece estar sendo amplamente utilizado pelos cirurgiões-dentistas em suas clínicas particulares. Apesar dos inúmeros benefícios, esse exame utiliza a radiação ionizante, que oferece um risco à saúde dos pacientes, trabalhadores e público em geral. Considerando a expansão do uso das radiações ionizantes na área da saúde, se faz necessária uma política de proteção radiológica em radiodiagnóstico. O cirurgiãodentista tem o dever de aumentar a qualidade do exame radiográfico, minimizando o risco atribuído à exposição radiográfica, observando o conceito "ALARA" (As Low As Reasonably Achievable), que preconiza uma exposição radiográfica com a menor dose possível de radiação. Estudos têm indicado que as recomendações para redução da dose, no exame radiográfico odontológico, não estão sendo seguidas pelos cirurgiões-dentistas em suas clínicas particulares (Tosoni, Campos \& Silva, 2013).

Desde a descoberta dos raios X por Wilhelm Conrad Röntgen, em novembro de 1895, várias pesquisas vêm enaltecendo a importância desta radiação para a humanidade, bem como suas ações deletérias devido à ação ionizante. Em nosso organismo, a ação ionizante se faz sentir especialmente nos cromossomos, cujos efeitos se manifestam durante a divisão celular, causando a evolução anormal ou morte da célula, sendo os principais riscos associados com baixas doses de radiação: o câncer, as mutações e as anomalias congênitas. As autoridades afirmam que qualquer dose de radiação tem potencial de produzir danos, mas, de acordo com a literatura, os efeitos potenciais da radiação advinda de aparelhos de raios $\mathrm{X}$ odontológico são mínimos, sendo o risco de indução de um câncer fatal por uma radiografia panorâmica de 1:1.000.000 e por uma radiografia intra-oral de 1:10.000.000. Todavia, apesar de o risco ser muito baixo, é importante considerar que os efeitos da radiação podem ser acumulativos; por isso, todos os dentistas têm a responsabilidade profissional com seus pacientes, sua equipe e a si mesmo de minimizar todos os riscos que possam estar associados à radiação. A conscientização dessa ação deletéria dos raios $\mathrm{X}$, através de efeitos somáticos ou genéticos, levou os pesquisadores a buscar medidas de segurança na aplicação desta radiação, surgindo na Alemanha em 1913 as primeiras regras para o uso dos raios X. Oito anos depois, em 1921, foram relatadas as primeiras recomendações de proteção radiológica pelo "British X-ray and Radium Committee". Todavia, foi somente em 1928 que foram estabelecidas as primeiras normas de proteção pela "International Commission of Radiological Protection" (Melo \& Melo, 2008).

Matheus, Montebelo Filho, Tanaka e Barros (2000), Kreich, Queiroz e Sloniak (2002) e Queiroga, Moreno, Figueiredo e Brasileiro (2010) relataram que a periodicidade dos erros nos exames radiográficos executados pelos graduandos de odontologia é maior que 80\%. Comparativamente, Carvalho et al. (2009) descreveu uma incidência de erros radiológicos que se aproximavam de 50\%. Entre os erros mais comuns nessas pesquisas, estão a posição errônea do filme, estriações, problemas no processamento, bem como na angulação horizontal ou vertical. Ainda não existe um acordo na literatura sobre qual é o erro mais comum, muito embora é sabido que os erros na técnica e execução da tomada radiográfica sejam mais corriqueiros que os erros na etapa de processamento.

Segundo Sissino e Moreira (2005) um dos principais objetivos dos profissionais que atuam na área de saúde deve ser oferecer condições que propiciem um atendimento de qualidade a seus pacientes. Em Odontologia, é sabido que, durante o processo de atendimento, diferentes materiais são utilizados, podendo gerar efluentes líquidos que precisarão de tratamento específico e uma grande variedade de resíduos sólidos que necessitarão de um gerenciamento adequado previamente a seu descarte, pois, constituem-se importantes fontes de contaminação para o ambiente e para população em geral.

Por fim, pode-se sugerir como principais causas desses erros o posicionamento inadequado do paciente e do aparelho de raios $\mathrm{X}$, radiografias que passaram pouco ou muito tempo em contato com as soluções reveladoras, manuseio inadequado da película após a revelação, armazenamento inapropriado, além de soluções com tempo de uso prolongado indicando esgotamento químico. 
$\mathrm{Na}$ contemporaneidade, com os avanços das tecnologias e dos sistemas informatizados, o surgimento da radiografia digital vem ocupando o lugar e em muitos casos até substituindo o uso de películas radiográficas e consequentemente de soluções químicas durante a etapa de processamento (Haiter Neto \& Melo, 2010). Outra vantagem desse recurso inovador é que as radiografias digitais fornecem uma maior qualidade da imagem formada ao mesmo passo que utiliza doses mais baixas de radiação durante a tomada radiográfica (Tsesis et al., 2008).

Outro benefício da radiografia digital é a facilidade de salvar e armazenar os dados no computador favorecendo a troca e envio de informações entre profissionais, além de possibilitar que o profissional efetue alterações, como por exemplo: ajustes de brilho, contraste, inversão da escala de cinza e aumento de aréas específicas (Candeiro, Bringel \& Vale, 2009; Braga, Silva, Oliveira, Júnior \& Marques, 2011). Sem falar que as radiografias digitais possuem a vantagem de não se deteriorarem, nem envelhecerem e não perderem a qualidade com o passar do tempo (Duarte, Araújo \& Sannomiya, 2008; Haiter Neto \& Melo, 2010).

De acordo com Iacopino (2007) a maior parte dos estudantes universitários egressos tem tendência a incorporar a sua prática clínica as tecnologias e protocolos aos quais foram expostos durante a sua vivência acadêmica. Porém, para que o uso desse recurso digital possa ser mais efetivo é necessário que durante a graduação os acadêmicos tenham a possibilidade de interação com essas tecnologias que, na maioria das vezes, são de alto custo para as universidades e que devido a falta de recursos financeiros apresentam uma certa resistência inicial de aquisição dos equipamentos digitais, sendo importante divulgar as vantagens proporcionadas por esses sistemas digitais, juntamente com a demonstração da economia proporcionada no decorrer do tempo por dispensar a reposição da película radiográfica e as etapas de processamento químico, além da aquisição rápida de imagens e consequente atendimento de um maior número de pacientes (Haiter Neto \& Melo, 2010).

\section{Considerações Finais}

O presente estudo teve enquanto relevância social e acadêmica, o intuito de apontar os principais erros cometidos pelos alunos do curso de Odontologia nas tomadas radiográficas e informar as possíveis causas e origens seja com relação ao posicionamento do paciente, a aplicação de técnicas ou modo de processamento dos filmes. Dessa maneira, ele poderá contribuir para a diminuição do número de exposições desnecessárias do paciente, pois através de um ponto de vista da ética odontológica tem-se que o cirurgião-dentista possui o dever de aumentar a qualidade das etapas de execução e do exame radiográfico propriamente dito, minimizando o risco atribuído à exposição radiográfica, observando o conceito ALARA (As Low As Reasonably Achievable), que recomenda uma exposição radiográfica com a menor dose possível de radiação.

Além de ser de grande interesse econômico para as intituições de ensino superior tendo em vista que a partir da análise dos dados pode-se traçar um perfil e melhorar a conduta da prática colaborando para a diminuição do desperdício e economia tanto de energia de operação de equipamentos de Raios-X quanto de material, como por exemplo: películas radiográficas, soluções reveladoras e fixadoras.

A pesquisa também desempenha grande importância ambiental, pois contribui para o gerenciamento inteligente de resíduos radiográficos que tem como objetivo minimizar sua produção e direcioná-lo para um encaminhamento seguro com eventual descarte apropriado.

Como sugestão para o desenvolvimento de trabalhos futuros os pesquisadores devem buscar aumentar a coleta e o tamanho da amostra (maior número de radiografias), quais as especialidades odontológicas funcionavam nas respectivas clínicas que foram coletadas as radiografias e, se possível, especificar quais os períodos do curso que atuavam nessas clínicas. Isso proporcionará dados mais precisos com relação aos fatores que induzem aos erros radiográficos, podendo-se traçar um panorama e possivelmente prever em qual período e especialidade do curso de odontologia os alunos cometerão mais erros radiográficos. 


\section{Agradecimentos}

Os autores agradecem à Universidade Federal de Campina Grande - UFCG e à toda equipe pela árdua colaboração no desenvolvimento deste trabalho e pesquisa científica. Agradecimento especial às professoras Luciana, Elizandra, Manuella e Kaline que foram essenciais nesse processo, por todo apoio e confiança durante a realização desta pesquisa. Por fim deixamos a reflexão de que, ás vezes, "dar errado foi a melhor coisa que poderia ter acontecido", pois é a partir dos erros que se pode aprender mais e buscar soluções que venham a contribuir com melhorias no ambiente acadêmico-científico e na sociedade.

\section{Referências}

Antunes, R. S. (2017). Resíduos de radiografias: Recolha e tratamento. 2017. 51 f. Dissertação (Mestrado) - Curso de Engenharia Química e Biológica, Centro de Ciências e Tecnologia, Universidade Nova de Lisboa.

Braga, E. F. A., Silva, P. G., Oliveira, P. T. V., Júnior, J. P. F., \& Marques, J. (2011). Comparação das imagens radiográficas digitais e convencionais em reaborções ósseas periodontais. Pesq Bras Odontoped Clin Integr, 11(4), 585-91.

Candeiro, G. T. D. M., Bringel, A. D. S. F., \& Vale, I. S. D. (2009). Radiologia digital: Revisão de literatura. Rev. Odontol. Araçatuba (Online), 30(2), 38-44.

Carvalho, P. L., Neves, A. C. C., Medeiros, J. M. F., Zollner, N. A., Rosa, L. C. L., \& Almeida E. T. D. C. (2009). Erros técnicos nas radiografias intrabucais realizadas por alunos de graduação. $R G O, 57(2), 151-155$.

Duarte, H. E. M., Araújo, C. C. M., \& Sannomiya, E. K. (2008). Sistemas digitais na Radiologia Dento-Maxilo-Facial: uma nova fase na Odontologia? Revista Brasileira de Odontologia, 65(1), 42.

Haiter Neto F., \& Melo D. P. (2010). Radiologia digital. Rev ABRO, 11(1), 5-17.

Iacopino A. (2007). The influence of "new science" on dental education: current concepts, trends, and models for the future. J Dent Educ., 71(4), 450-22.

Kreich, E. M., Queiroz, M. G. S., \& Sloniak, M. C. (2002). Controle de qualidade em radiografias periapicais obtidas no curso de Odontologia da UEPG. Publicatio UEPG: Ciências Biológicas e da Saúde, 8(1), 33-45.

Matheus, R. A., Montebelo Filho, A., Tanaka, E. E., \& Barros, R. M. P. (2000). Avaliação no desempenho de acadêmicos durante tomadas radiográficas periapicais, pela técnica da bissetriz, realizadas na Faculdade de Odontologia da Universidade Norte do Paraná (Unopar). Revista da Faculdade de Odontologia - UPF, 5(2), 25-29.

Melo, M. D. F. B., \& Melo, S. L. S. (2008). Condições de radioproteção dos consultórios odontológicos. Ciência \& Saúde Coletiva, 13, $2163-2170$.

Pereira, A. S., Shitsuka, D. M., Parreira, F. J., \& Shitsuka, R. (2018). Metodologia da pesquisa científica. Ed. UAB/NTE/UFSM.

Queiroga, M. A. S., Moreno, N. P. P., Figueiredo, C. B. O., \& Brasileiro, C. B. (2010). Avaliação dos erros radiográficos cometidos por graduandos de odontologia em técnicas radiográficas intrabucais. Arquivos em Odontologia, 46(2), 61-65.

Silva, J. M. F., Oliveira, L. C., Daroz, B. G., Peyneau, P. D., Pereira, T. C. R., \& Azevedo-Vaz, S. L. (2016). Erros cometidos por estudantes de Odontologia de uma universidade pública brasileira na realização de radiografias periapicais. Revista da ABENO, 16(1), 99-109.

Sisinno, C. L. S., \& Moreira, J. C. (2005). Ecoeficiência: um instrumento para a redução da geração de resíduos e desperdícios em estabelecimentos de saúde. Cadernos de saúde pública, 21(6), 1893-1900.

Tosoni, G. M., Campos, D. M., \& Silva, M. D. (2013). Frequência de cirurgiões-dentistas que realizam exame radiográfico intrabucal e avaliação das condições para a qualidade do exame. Revista de Odontologia da UNESP, 32(1), 25-29.

Tsesis, I., Kamburoglu, K., Katz, A., Tamse, A., Kaffe, I., \& Kfir A. (2008). Comparison of digital with conventional radiography in detection of vertical root fractures in endodontically treated maxillary premolars: an ex vivo study. Oral Surg Oral Med Oral Pathol Oral Radiol Endod, 106(1), 124-128. 